\title{
AVALIAÇÃO PRELIMINAR DOS CUSTOS DE RESFRIAMENTO DE LARANJA IN NATURA ${ }^{1}$
}

\author{
BÁRBARA TERUEL²; LUÍS CORTEZ3; PAULO LEAL ${ }^{3}$, LINCOLN NEVES FO ${ }^{4}$
}

\begin{abstract}
RESUMO - Este trabalho apresenta um estudo para determinar os custos do resfriamento de laranja Valência (Citrus Sinensis Osbeck) num sistema com ar forçado $\left(\mathrm{Ta}=1^{\circ} \mathrm{C}\right.$ e UR $\left.=88,4 \pm 2,0 \%\right)$ e numa câmara convencional. Os frutos foram resfriados condicionados em dois tipos de embalagens, com área efetiva de abertura de 3,5\% e 40\%. O custo total anual foi determinado com o cálculo dos custos fixos e variáveis da instalação, e o custo total de resfriamento, baseado na quantidade de frutos que podem ser resfriados em cada sistema, num mesmo período. Comprovou-se que o custo de resfriamento chega a ser de $20 \%$ a $40 \%$ menor quando os frutos são resfriados no sistema com ar forçado, se comparado com o custo quando os frutos foram resfriados no sistema convencional.
\end{abstract}

Termos para indexação: Citrus Sinensis Osbeck, tempo de resfriamento, embalagem, ar forçado, câmara de estocagem

\section{PRELIMINARY EVALUATION OF COOLING COST OF FRESH ORANGE}

\begin{abstract}
This paper presents study of the cooling cost of Valencia orange in a forced-air system and room cooling $\left(\mathrm{Ta}=1^{\circ} \mathrm{C}\right.$ and $\mathrm{RH}=88,4 \pm 2,0 \%$ ). The fruits was conditioned in two package with $3,5 \%$ and $40 \%$ of opening effective area. The annual total cost was determined with the fixed and variable costs of the installation and the cooling total costs with the quantity of cooling products in the each system for the same period. The cooling total cost is the 40 to $20 \%$ smaller, if compared with the cost when the fruits cooling in the conventional room.
\end{abstract}

Index terms: Citrus Sinensis Osbeck, cooling time, package, forced-air cooling, room cooling

\section{INTRODUÇÃO}

Um aspecto a ser considerado quando se realiza resfriamento são os custos, que envolvem uma série de parâmetros, dependendo do sistema em particular. Alguns estudos da área têm demonstrado que os sistemas de resfriamento com ar forçado são mais econômicos (menor custo e consumo energético), se comparados com os sistemas de resfriamento com água gelada e câmaras de estocagem convencionais (Kader, 1992; ASRHAE, 1994).

Os custos envolvendo os sistemas de resfriamento com ar forçado podem ser menores se utilizados unicamente para o resfriamento rápido dos produtos, para que possam ser estocados em câmaras de resfriamento e transportados e comercializados (Fraser, 1991; Fraser \& Otten, 1992). Entre os principais fatores que afetam os custos de resfriamento, encontram-se: a quantidade de horas de operação, a temperatura do meio de resfriamento, tipos de embalagem, entre outros.

$\mathrm{Na}$ América do Norte, particularmente na Flórida, onde a produção de frutas cítricas é grande, o resfriamento de laranja in natura é muito usado, onde já vários trabalhos foram desenvolvidos para avaliar o comportamento dos custos envolvendo o resfriamento, quando usados os diferentes sistemas e embalagens.

A título de exemplo, Gaffney \& Bowman (1971) desenvolveram um estudo econômico para avaliar os custos do resfriamento de frutas cítricas, num sistema com água gelada e num sistema com ar forçado. As frutas foram resfriadas em caixas de papelão e a granel. Estes autores comprovaram que, quando as frutas foram resfriadas no sistema de resfriamento com ar forçado (a granel, em caixas de papelão e em palhetes), o custo total variou de US\$ 0,53 a 0,62 por $\mathrm{kg}$ de produto. Quando feito o resfriamento dos frutos no sistema com água gelada, o custo de resfriamento foi de U\$ 0,43 por $\mathrm{kg}$ de produto. Esta diferença de preços, provavelmente, é devida a que os sistemas de resfriamento com água garantem tempos de resfriamento bem menores, se comparado com sistemas que utilizam ar forçado.

Por outro lado, Baird et. al. (1985) resfriaram laranja a granel num sistema com ar forçado, condicionando os frutos em embalagens de papelão com área de abertura entre 1 e $5 \%$. Os autores comprovaram que o custo de resfriamento oscilou entre US\$ 0,46 e US\$ 0,62 (por kg de produto) para o resfriamento a granel. Quando os frutos foram resfriados nas caixas de papelão, o custo do resfriamento oscilou entre US $\$ 0,50$ e US\$ 0,95 por $\mathrm{kg}$ de produto.

Já Baird et al., em 1988, desenvolveram um modelo econômico para estudar a influência de diferentes fatores na taxa de resfriamento e no custo total, para produtos resfriados num sistema com ar forçado, a granel e em embalagens de papelão. $\mathrm{O}$ custo de resfriamento para os produtos resfriados a granel foi de US\$ 0,10 a US\$ 0,14 por $\mathrm{kg}$ de produto. Quando realizado o resfriamento em embalagens com $9 \%$ de área de abertura (tempo

1 (Trabalho 079/2001). Recebido: 28/03/2001. Aceito para publicação: 04/10/2001.

2 UNICAMP - FEM, Área Interdisciplinar de Planejamento de Sistemas Energéticos, C. Postal. 6122, 13081-970. Campinas - SP

3 UNICAMP - FEAGRI, Depto de Construções Rurais, C. Postal 6011.

4 UNICAMP - FEA, Depto de Engenharia dos Alimentos, C. Postal 6010. 
de resfriamento de $3 \mathrm{~h} 5$ ), o custo foi de US\$ 0,43 por $\mathrm{kg}$. Nas embalagens com $3 \%$ de área efetiva (tempo de resfriamento de 4 h), o custo foi de US\$ 0,22 por kg de produto.

Particularmente no Brasil, o resfriamento rápido de laranja in natura não é utilizado. Apesar de o País ser um dos maiores produtores mundiais destas frutas, existe um grande desconhecimento quanto aos custos envolvidos no resfriamento.

Neste trabalho, objetivou-se determinar o custo de resfriamento de laranja in natura, resfriada num sistema com ar forçado, comparando-se com o custo quando resfriadas num sistema convencional. Os frutos tinham sido condicionados em dois tipos de embalagens, diferentes quanto à área efetiva de abertura para o passo do ar resfriado. Com a avaliação preliminar que está sendo apresentada neste trabalho, pretendem-se mostrar índices práticos que incentivem, gradualmente, a implantação de sistemas de resfriamento rápido, por parte dos produtores.

\section{MATERIAL E MÉTODOS}

\section{Cálculo econômico}

A metodologia utilizada para desenvolver o cálculo econômico, foi baseada no software CUSTAGRI, desenvolvido pelo Instituto de Economia Agrícola (IEA), em parceria com o Centro Nacional de Pesquisa em Informática para a Agricultura (Kaplan, 1983; CNPTIA/EMBRAPA, 1997).

\section{Custo total (CT)}

O cálculo do custo total anual (Equação 1) foi feito a partir do cálculo dos custos fixos em função da depreciação (D) e da taxa de juros $(\mathrm{J})$. Os custos variáveis ou operacionais foram determinados em função dos custos de manutenção $\left(\mathrm{R}_{\mathrm{m}}\right)$, do custo da energia (E) e da mão-de-obra $\left(\mathrm{M}_{\mathrm{O}}\right)$. As informações utilizadas foram fornecidas pelos fabricantes dos equipamentos e agências de financiamento, e outros dados foram obtidos experimentalmente.

$$
\mathrm{CT}=\mathrm{Cf}(\mathrm{D}+\mathrm{J})+\mathrm{Cv}\left(\mathrm{R}_{\mathrm{m}}+\mathrm{E}+\mathrm{M}_{\mathrm{o}}\right)
$$

$\mathrm{O}$ custo inicial $(\mathrm{Ci})$ da câmara convencional é de R\$ 31330,00 (dados fornecidos pela RECROSUL, 1999), e do sistema de resfriamento com ar forçado de $\mathrm{R} \$ 32641,00$ (dados fornecidos pela TORIN Ventiladores, 1999). Considerou-se um valor de $10 \%$ para o cálculo do valor final presumido, com um período de vida útil de 25 anos (câmara convencional) e 20 anos (sistema de ar forçado). Um total de horas de funcionamento do sistema de refrigeração (h) de $2160 \mathrm{~h}$ (18 horas/dia x 120 dias/ano (20 dias por mês, 6 meses de safra)). A taxa anual de juros (J) considerada foi de $12 \%$ a.a. (Agência Especial de Financiamento Industrial (FINAME), janeiro a março/2000).

Para o cálculo dos custos variáveis, foi considerada uma porcentagem de manutenção de $0,5 \%$ a.a., com uma jornada de trabalho de 8 horas, 24 dias por mês e um salário, para o operador, de $\mathrm{R} \$ 200,00$ ao mês. O consumo de energia de ambos os sistemas foi determinado experimentalmente, sendo de $4,0 \mathrm{kWh}$ (sistema convencional), e de 5,3 $\mathrm{kWh}$ (sistema com ar forçado) (Teruel et al., 1999). O preço médio do kwh foi de $\mathrm{R} \$ 0,11 / \mathrm{kWh}$ (preço médio do Kwh comercial, na UNICAMP, no período de agosto 1999 a 2000).

\section{Custo total de resfriamento (CTP)}

O custo total de resfriamento (CTP) foi obtido dividindo o custo total anual (CT) entre a quantidade de produto resfriado (M) (Equação 2):

$$
C T P=\frac{C T}{M}
$$

$\mathrm{O}$ valor do CTP pode ser expresso em unidade monetária por caixa (R $\$ / \mathrm{cx}(\mathrm{US} \$ / \mathrm{cx}))$, ou por unidade de peso (R $\$ / \mathrm{kg}$ (US\$/ $\mathrm{kg})$ ). Para determinar CTP, considerou-se o tempo médio que foi necessário para atingir o tempo dos sete oitavos do resfriamento, determinado de forma experimental em pesquisas precedentes (Teruel et al., 1999; Teruel, 2000). Este tempo refere-se ao tempo necessário para que o produto atinja uma temperatura, aproximadamente, no centro do mesmo, que seja equivalente a $1 / 8$ da diferença entre a temperatura inicial do produto e a temperatura do meio de resfriamento.

O tempo de resfriamento foi obtido quando os frutos foram condicionados em dois tipos de embalagens, que diferiam com respeito à área livre de passo do resfriado. O primeiro tipo de embalagem possuía $40 \%$ de área efetiva para a passagem do ar (EI), em embalagens comumente usadas para o transporte dos produtos até o galpão de embalagem. Esta embalagem é plástica e tem uma capacidade de até $25 \mathrm{~kg}$.

O segundo tipo, denominado como EII, com 3,5\% de área efetiva, são as embalagens de papelão usadas no Brasil para a comercialização das frutas. Estas embalagens podem receber até $20 \mathrm{~kg}$ de frutos.

Os frutos foram resfriados num sistema com ar forçado, montado no interior de uma câmara de resfriamento, adicionando como principal elemento de consumo energético um ventilador centrífugo. Este ventilador tem a função de forçar o ar resfriado a passar transversalmente, a maiores velocidades do que na câmara convencional, entre o volume de caixas que se encontram no interior do túnel, fechado com uma lona. Quando usada a câmara convencional para o resfriamento, o ar circula a menores velocidades por entre os produtos, o que se traduz em maiores tempos de resfriamento.

A temperatura do ar de resfriamento foi de $1{ }^{\circ} \mathrm{C}$, e considerando que a temperatura inicial dos frutos foi em média de $26^{\circ} \mathrm{C}$, estes atingiram a condição de resfriamento (tempo dos sete oitavos do resfriamento) quando, no centro dos mesmos, a temperatura foi de aproximadamente $3^{\circ} \mathrm{C}$. No interior da câmara de resfriamento, foram colocadas 12 caixas de laranja de aproximadamente $20 \mathrm{~kg}$ cada uma. Para estas condições, o tempo de resfriamento, obtido de forma experimental em cada sistema, é mostrado a seguir (Teruel, 2000):

\section{- Câmara convencional (estocagem)}

- Resfriamento em caixas EI (40\% área de aberturas): 6 horas.

- Resfriamento em caixas EII (3,5\% área de aberturas): 18 horas

- Sistema de resfriamento com ar forçado - Resfriamento nas embalagens EI: 3 horas

- Resfriamento nas embalagens EII: 5 horas

Considerando que, de cada vez, sejam colocadas aproximadamente 80 caixas no interior da câmara, quando é feito o resfriamento nas caixas EI ( $40 \%$ área efetiva) o tempo de resfriamento é de 6 horas (câmara de estocagem). Desta forma, num dia, poderão ser resfriados, aproximadamente, dois lotes de caixas, totalizando 160 caixas por dia. Para um período de trabalho de 10 horas por dia, durante 20 dias ao mês (fins de semana, manutenção, ect.), e 6 meses de safra (120 dias ao ano), poderão 
ser resfriadas 19200 caixas/ano.

Quando o resfriamento é feito nas embalagens EII ( $3,5 \%$ de área efetiva), o tempo de resfriamento é 3 vezes maior, o que faz diminuir o fator de rotatividade da câmara, necessitando-se mais de um dia para resfriar um lote, podendo então ser resfriadas no mesmo período até 10.000 caixas.

No entanto, quando usado o sistema com ar forçado, a quantidade de caixas que podem ser resfriadas num mesmo período, aumenta sensivelmente, podendo-se resfriar até 3 lotes de 80 caixas, totalizando 28800 caixas (embalagens EI), e quando condicionados os produtos na embalagem EII, podem resfriarse até 19000 caixas.

\section{RESULTADOS E DISCUSSÃO}

\section{Custo total anual (CT)}

No sistema de resfriamento com ar forçado, devido à adição do ventilador, o consumo de energia apresenta um incremento de $25 \%$ com relação ao sistema de resfriamento na câmara convencional. O custo total por hora (CT), na câmara de estocagem, foi de $\mathrm{R} \$ 3,01 / \mathrm{h}$ e no sistema de resfriamento com ar forçado, de $\mathrm{R} \$ 3,60 / \mathrm{h}$; uma diferença de, aproximadamente, $16 \%$ (Tabela 1).

Na Figura 1, pode ser observado que, à medida que o tempo de resfriamento aumenta, o CT aumenta, seguindo uma relação linear para os dois tipos de sistemas de resfriamento (câmara estocagem, CE e sistema de ar forçado, CAF). Este aumento é sempre maior nos sistemas de resfriamento convencionais, pois o tempo de resfriamento, da mesma quantidade de produto, é maior que no sistema com ar forçado.

O elemento que tem maior influência no valor do CT, para cada sistema de resfriamento, é o consumo de energia. No câmara convencional utilizada neste trabalho, o custo da energia representa $14 \%$ do custo total, já no sistema de ar forçado, representa aproximadamente $17 \%$.

\section{Custo total de resfriamento (CTP)}

O CTP é consideravelmente maior, quando as frutas são acondicionadas nas caixas EII, que quando condicionadas em caixas EI. No sistema convencional, esta diferença representa $91 \%$, e quando os produtos são resfriados no sistema com ar forçado, esta diferença é de 52\% (Tabela 2). O tempo de resfriamento depende do tipo de embalagem usado, o que, por sua vez, define as possibilidades de rotatividade da câmara.

Mesmo que o CT do sistema com ar forçado seja $20 \%$ maior que o CT do sistema convencional, o CTP do sistema de ar forçado é de $20 \%$ a $37 \%$ menor, para embalagens com $40 \%$ e $3,5 \%$ de área efetiva, respectivamente, que quando os frutos são resfriados nestes mesmos tipos de embalagens no sistema convencional. Esta relação está diretamente relacionada com o tempo de resfriamento. Para um tempo de resfriamento menor, a rotatividade da câmara será maior, podendo ser resfriadas um maior número de caixas, no mesmo período.

Na Figura 2, mostra-se uma comparação do CTP para ambos os sistemas de resfriamento, usando a embalagem EI. Como se pode observar, comparando esta figura com a anterior (Figura 1), independentemente de que o CT seja maior no sistema de ar forçado, o CTP é menor. Isto é explicado porque a rotatividade dos sistemas de resfriamento com ar forçado é maior, o que se traduz num maior número de caixas resfriadas num mesmo período, e, portanto, faz diminuir o custo total de resfriamento por caixa. Nesse ponto, os sistemas de resfriamento com ar forçado tornam-se muito mais eficientes, economicamente, se comparados com os sistemas convencionais.

No cálculo do custo da energia, foi considerado o preço que a UNICAMP paga (R $\$ 0,11 / \mathrm{kWh}$ ), que geralmente é mais baixo que o preço do $\mathrm{kWh}$ industrial. Se fosse considerado o preço do $\mathrm{kWh}$ industrial médio em julho de 1999 ( $\mathrm{R} \$ 0,19 / \mathrm{kWh})$, o valor de CTP aumentaria de $\mathrm{R} \$ 0,34 / \mathrm{cx}$ para $\mathrm{R} \$ 0,36 / \mathrm{cx}$ (EI); e de $\mathrm{R} \$ 0,65 / \mathrm{cx}$ para $\mathrm{R} \$ 0,69 / \mathrm{cx}(\mathrm{EII})$. Estes valores representam um acréscimo de aproximadamente $8 \%$ no sistema convencional. Para o sistema com ar forçado, o CTP aumentaria de R 0,27 /cx para $\mathrm{R} \$ 0,29 / \mathrm{cx}(\mathrm{EI})$, e de R 0,41 /cx para $\mathrm{R} \$ 0,44$ /cx (EII). O que representa um acréscimo no CTP na ordem de $6 \%$. Nota-se que o custo do $\mathrm{kWh}$ influencia notavelmente o valor do CTP.

Por outro lado, para o cálculo da quantidade de caixas a serem resfriadas, considerou-se que a câmara de resfriamento seria utilizada em todo o seu volume. É necessário ressaltar que o CTP dependerá, em grande medida, da rotatividade da câmara em função do tempo de resfriamento. Por esta razão, se for maior a quantidade de caixas resfriadas, menor será o CTP e vice-versa.

A título de comparação, se fossem resfriadas até 15000 caixas na câmara de estocagem, o custo de resfriamento subiria de $R \$ 0,34 / \mathrm{cx}$ para $\mathrm{R} \$ 0,44 / \mathrm{cx}$. No sistema de resfriamento com ar forçado, no mesmo período, resfriando-se até 23000 caixas, o CTP aumentaria de $\mathrm{R} \$ 0,27 / \mathrm{cx}$ para $\mathrm{R} \$ 0,35 / \mathrm{cx}$; o que representa um aumento do custo de aproximadamente $30 \%$ (Teruel, 2000).

\section{Custo incrementado às caixas de laranja por conceito de resfriamento}

Na Tabela 2, apresenta-se um resumo do CTP e do acréscimo que este incorpora ao custo da caixa de laranja que é produzida na Fazenda Sete Lagoas, em SP, para exportação. As frutas são selecionadas no packinghouse e submetidas a tratamento de lavagem, encerado e secagem.

Observa-se que o custo incrementado por conceito de resfriamento representa de 5 a $7 \%$ de acréscimo, com respeito ao custo da caixa sem resfriamento, quando usado um sistema de resfriamento com ar forçado. Já, quando o resfriamento é realizado em câmaras convencionais, onde o tempo de resfriamento é maior, o acréscimo pode chegar a $11 \%$, um valor até 2 vezes maior que quando realizado o resfriamento num sistema com ar forçado.

De igual forma, quando usadas as embalagens com maior área efetiva de abertura para a passagem do ar resfriado (EI, $40 \%$ área efetiva), o custo total da caixa resfriada chega a ser de $35 \%$ a $45 \%$ menor, se comparado com a embalagem EII (3,5\% área efetiva).

Particular importância tem, para a eficiência do resfriamento e diminuição do custos, o tipo de embalagem onde são condicionados os produtos, pois a maior área de abertura permite que estes atinjam a condição de resfriamento em menor tempo, garantindo uma vida de prateleira maior e maior qualidade.

Este trabalho apresenta apenas um estudo preliminar, onde foram feitas uma série de considerações e simplificações para facilitar os cálculos; por esta razão, os valores e índices mostrados são aproximados. Para cada situação em particular, devem-se considerar as condições específicas. 


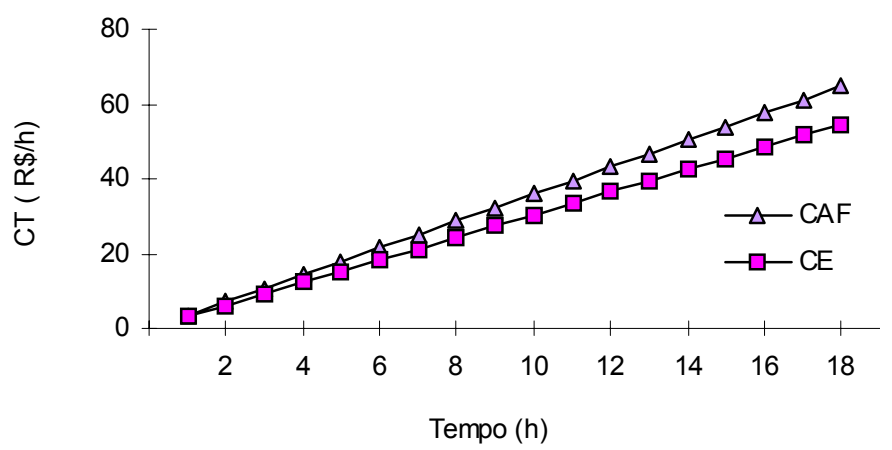

FIGURA 1- Custo total em função do tempo de resfriamento. CEcâmara de estocagem; CAF- câmara de ar forçado.

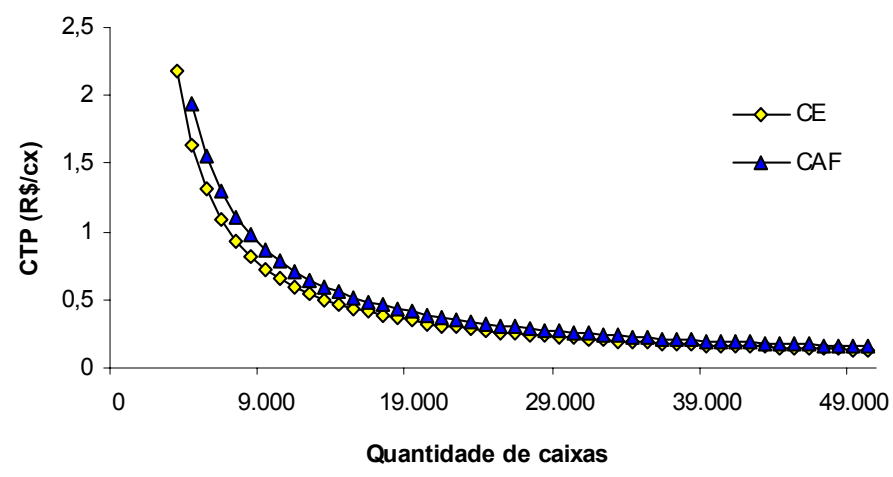

FIGURA 2- Custo de resfriamento em função do número de caixas resfriadas. CE- câmara de estocagem; CAF- câmara de ar forçado.

TABELA 1- Custo anual dos sistemas de resfriamento.

\begin{tabular}{|c|c|c|c|c|c|c|}
\hline \multirow[t]{2}{*}{$S$ is te $m$ a } & \multicolumn{2}{|c|}{ 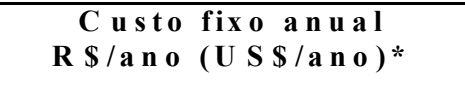 } & \multicolumn{3}{|c|}{$\begin{array}{c}\text { Custo variável anual } \\
\text { R \$/ano(US \$/ano) }\end{array}$} & 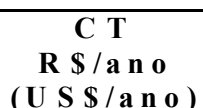 \\
\hline & $\begin{array}{c}\text { Depreciação } \\
\text { (D) }\end{array}$ & $\begin{array}{c}\text { J u ros } \\
(\mathrm{J})\end{array}$ & $\begin{array}{c}\text { M a nu ten çã o } \\
(\mathrm{R} m)\end{array}$ & 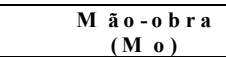 & $\begin{array}{c}\text { Energia } \\
\text { (E) }\end{array}$ & \\
\hline
\end{tabular}

*O valor da taxa cambial assumida (R \$US\$ 1,75), para a transformação em US\$, corresponde ao valor médio de fevereiro a março/2000 (Fundação Instituto de Pesquisas Econômicas, FIPE).

D- depreciação; J- juros; m- manutenção; Mo- mão-de-obra; E- custos da energia consumida; Cf- custos fixos; Cv- custos variáveis.

TABELA 2- Custo total de resfriamento e incremento ao custo de produção por caixa de laranja.

\begin{tabular}{|c|c|c|c|c|c|c|}
\hline \multirow[t]{2}{*}{$\mathrm{S}$ is te $\mathrm{m}$ a } & \multicolumn{2}{|c|}{$\mathbf{C} \mathbf{T} \mathbf{P}, \mathbf{R} \$ / \mathbf{c} x$} & \multicolumn{2}{|c|}{$\begin{array}{c}\text { Custodacaixa } \\
\text { produzida* }+ \text { C T P }\end{array}$} & \multicolumn{2}{|c|}{ A crés cimo, \% } \\
\hline & E I & E I I & E I & E I I & E I & E I I \\
\hline $\begin{array}{l}\text { Câm ara de } \\
\text { e stocage m }\end{array}$ & 0,34 & 0,65 & 5,19 & 5,50 & 6,5 & 11,8 \\
\hline A r forçado & 0,27 & 0,41 & 5,12 & 5,26 & 5,2 & 7,8 \\
\hline
\end{tabular}

*O custo total médio (dos últimos 5 anos) de uma caixa de laranja (20 kg), na Fazenda Sete Lagoas, foi de aproximadamente R\$ 4,85 (Visita Técnica, 1999).

\section{CONCLUSÕES}

Embora o custo total seja 19\% maior nos sistemas com ar forçado, o custo de resfriamento é até $40 \%$ menor, se comparado com o sistema convencional. De igual forma ,o custo de resfriamento é de $21 \%$ a $37 \%$ menor, quando os frutos são resfriados no sistema com ar forçado, nas embalagens EI e EII, respectivamente, se comparado com o custo quando foram resfriados nas mesmas embalagens no sistema convencional.

Os valores de custos que estão sendo apresentados neste trabalho, formam parte de um estudo preliminar; portanto, devem ser usados com cautela em casos particulares.

Atendendo aos resultados do trabalho, recomenda-se a implementação de sistemas com ar forçado, para o resfriamento rápido de frutas após a colheita por apresentarem as seguintes vantagens: altas taxas de resfriamento, simplicidade de operação e baixos custos.

\section{AGRADECIMENTOS}

À FAPESP, pelo apoio financeiro.

\section{REFERÊNCIAS BIBLIOGRÁFICAS}

ASHRAE. Commodity Storage Requeriments. Refrigeration systems and applications handbook. Atlanta: American Society of Heating, Refrigerating and Air-Conditioning Engineers, 1994. BAIRD, C. D.; CHAU, K. V.; GAFFNEY, J. J. An engineering/ 
economic model for evaluating forced-air cooling systems for fruits and vegetables. Refrigeration Science and Technology. Institute International of Refrigeration, 1985. p. 259-266.

BAIRD, C. D.; GAFFNEY, J. J.; TALBOT, M. T. Desing criteria for efficient and cost effective forced-air cooling systems for fruits and vegetables. ASHRAE Transactions, v. 94, p. 1434-1453, 1988.

CNPTIA/EMBRAPA. Centro Nacional de Pesquisa Tecnológica em Informática para a Agricultura Instituto de Economia Agrícola (IEA/SSA). Sistema CUSTRAGRI. Sistema Integrado de Custos Agropecuários. Versão 1.0. 1997. 75 p .

FRASER, H. W. Forced-air rapid cooling of fresh Ontario fruits and vegetables. Toronto: Ministry of Agriculture and Food. AGDEX 202-736. 1991.4p.

FRASER H.; OTTEN, L. Predicting $7 / 8$ cooling times for peaches by comparing heat transfer modelling and field measurement methods. American Society of Agricultural Engineers, 1992. 10p. (Paper no. 92-6016.).

GAFFNEY, J.J.; BOWMAN, E. K. An enginnering/economic evaluation of different concepts for precooling citrus fruits. ASHRAE. Symposium Bulletin. 4-70. USA: Departament
Agriculture, 1971.

KADER, A.A. Postharvest technology of horticultural crops. 2.ed. Davis: University of California, 1992. 295p.

KAPLAN, S. Energy economics: quantitative methods for energy and environmental decisions. New York: Polytechnic Institute, McGraw-Hill. 1983.352p.

TERUEL M. B. J; CORTEZ, L. A. B.; LEAL, P. M.; LIMA, G. A B. Obtenção numérico/experimental das curvas de resfriamento de laranja (variedade Valência). In: CONGRESSO BRASILEIRO DE ENGENHARIA MECÂNICA. 12. 1999. Anais...

TERUEL M. B. J; CORTEZ, L. A. B.; LEAL, P. M.; NEVES FO, C. L. Curvas de enfriamiento de naranjas refrigeradas en un sistema con aire forzado. Revista Información Tecnológica, v. 11, n. 4, p. 25-29, 2000.

TERUEL, M. B. J. Estudo teórico-experimental do resfriamento com ar forçado de laranja e banana. 2000.300 p. Tese (Doutorado em Planejamento de Sistemas Energéticos)-Universidade Estadual de Campinas, Faculdade de Engenharia Mecânica, Campinas, 2000. 